\title{
Comparative Study of Load Balancing Algorithms in Cloud Environment using Cloud Analyst
}

\author{
Veerawali Behal \\ Mtech(SS) Student \\ Department of Computer Science \& Engineering \\ Guru Nanak Dev University, Amritsar
}

\author{
Anil Kumar \\ Assistant Professor \\ Department of Computer Science \& Engineering \\ Guru Nanak Dev University, Amritsar
}

\begin{abstract}
This is age of internet. There is hardly any domain where internet do not play significant role. Cloud Computing relates to internet computing that has revolutionized the whole world. Cloud Computing has undeniably benefited the users in many ways. Load Balancing is emerging as the hottest issue in cloud environment at present. Load due to client requests must be balanced by resources of service provider. In this study we proposed a theoretical framework which is combination of load balancing policy and service broker policy for cloud environment. This paper includes the comparison of the results of proposed algorithm with existing algorithm .This study accomplish that there is extreme reduction in response time at client part and reduction in datacenter request servicing time and resources of resource providers are managed efficiently and effectively by using the proposed algorithm. Several parameters are also identified and these are utilized to compare the traditional round robin load balancing policy with proposed load balancing strategy by using cloud analyst tool as simulator.
\end{abstract}

\section{General Terms}

Cloud Environment, Load Balancing Algorithms.

\section{Keywords}

Cloud Computing, Cloud Analyst, Service Broker, Load balancing.

\section{INTRODUCTION}

Cloud Computing is probably the only technology that completely complements the internet. It is defined as new style of computing in which vigorously flexible and often virtualized resources are provided as a services over internet. There are several definitions for the cloud. The definition of cloud computing provided by "The National Institute of Standards and Technology" says that Cloud computing is a model for enabling convenient, on-demand network access to a shared pool of configurable computing resources that can be rapidly provisioned and released with minimal management effort or service provider interaction[1]. Cloud computing comes up with various services such as infrastructure as a service, platform as a service, and software as a service. Cloud computing is a recent technology in IT that shifts the desktop data to larger data centers [3]. In a distributed environment, clients generate request randomly in any processor. However, the major flaw of this randomness is associated with assignment of task. This causes load imbalance, and due to this some processor are overloaded and some of them are under loaded [4].The main target of load balancing is to transfer load from overloaded process to under loaded process explicitly. Cloud computing has been extensively selected by the business world, however there are number of issues like Load Balancing, Migration of Virtual Machine, Threats and opportunities of the cloud security, Sustainability, Privacy etc. that are not accepted [4].Load balancing is one of the main issue among the entire issues. Load balancing is a procedure to disseminate the load evenly to all the nodes in the integral cloud to achieve a high user satisfaction and resource utilization rate. Fair load balancing techniques helps in making business as per user satisfaction and also reduces response time [5] [6].

\subsection{Load Balancing \& Algorithms}

Load balancing is a necessary mechanism to increase SLA and better usage of resources. Load Balancing must consider two main tasks, one is the resource allocation and other is task scheduling in distributed environment. Efficient provisioning of resources and scheduling of resources as well as task will ensure resources are utilized efficiently under condition of high or low load, resources are easily available and cost of using resources is reduced [16].

\subsubsection{Virtual Machine Load Balancing} Algorithms for Cloud Computing [7]

Datacenters use the VM load balancer to balance load of requests between available virtual machines. There are three existing algorithms which are:

\subsubsection{Round Robin Algorithm (RR):}

Round Robin scheduling is one of the traditional approach .In it time slices are assigned to each process in equal fraction and in circular fashion. This is not priority-oriented scheduling algorithm, as each job is handled to available virtual machine in circular order. This leads to situation where some nodes are thickly loaded and some are moderately loaded. So this causes the load of the system to imbalance [5] [11].

\subsubsection{Throttled Algorithm:}

It works by allocating convenient virtual machine when client sends request to load balancer. Throttled load balancer takes responsibility of maintaining an index table of all virtual machines along with their states whether busy or available. At beginning, all virtual machines are set as available. When data center controller receives a new request, it inquires the balancer for next virtual machine allocation. Then balancer checks the table from start until a match of appropriate virtual machine is found or table is checked completely. If available virtual machine is found then the balancer returns the virtual machine id to data center controller, then data center controller send request to particular virtual machine recognized by that id. Data center controller then also sends 
notification to the balancer of new allocation so that it can update the allocation table .In case if virtual machine is not found, then the balancer returns -1 value and data center queues the request. When the virtual machine finishes with the processing of the allocated request then the data center controller receives the response cloudlet and then it sends the notification to balancer to virtual machine de-allocation [9][14].

\subsubsection{Active Monitoring Load Balancing:}

This Load balancing approach tends to maintain identical workloads on all the available Virtual machines. It works by allocating appropriate virtual machine when client sends request to load balancer. Active Monitoring load balancer takes responsibility of maintaining an index table of all virtual machines along with the number of requests presently allocated to each virtual machine. When data center controller receives a new request, it inquires the balancer for next virtual machine allocation. Then balancer checks the table from start until a match of appropriate least loaded virtual machine is found. If there is more than one least loaded virtual machine, then very first virtual machine is selected. The balancer returns the virtual machine id to data center controller, then data center controller send request to particular virtual machine recognized by that id. Data center controller then also sends notification to the balancer of new allocation so that it can update the table by increasing number of allocation count of that particular virtual machine. When the virtual machine finishes with the processing of the allocated request then the data center controller receives the response cloudlet and then it sends the notification to balancer to decrease the allocation count by one of that particular virtual machine in the table [9].

The paper is organized as follows. In Section 2, problem statement is given. Section 3, discusses some recent review of similar work in the cloud computing field. Section 4 discusses the proposed work. Section 5 describes the simulation setup and performance analysis and results. Section 6 concludes the paper.

\section{PROBLEM STATEMENT}

In order to perform load balancing, several algorithms have been proposed to combat this vexing issue. In Round Robin algorithm, load is transferred randomly that can cause some server to thickly loaded while other servers leads to idle or lightly loaded. Equally Load Distributing boost the system performance by relocating load from thickly loaded servers to lightly loaded servers.

The comparison analysis of various parameters such as response time and datacenter processing time has been done and performance of various algorithms is being analyzed.

\section{REVIEW OF RELATED WORK}

Several studies have been reported that have been focused on load balancing in cloud environment. In this section, numbers of papers are studied which relates to cloud computing and its central issue of load balancing.

Ram Prasad Padhy et al. describes the key concepts of cloud computing, services offered, its key constituents, functions provided by the cloud, and also reviewed some existing load balancing algorithms which can be practiced on clouds [2]. Y. Jadeja et al. have discussed the architectural design of cloud computing, its advantages and some issues such as security, privacy, reliability etc. and research challenges along with some of its applications [1]. Martin Randles et al. correlates the performance of three load balancing algorithms, discussed the shortcomings and investigated why it is not possible to have centralized scheduling policy in the cloud environment. Authors examined three feasible solutions proposed for load balancing, which are Honeybee Foraging Behavior algorithm, Random Sampling algorithm and Active Clustering algorithm [8]. Authors have reviewed that Cloud Analyst can be used to interpret a case study of a social networking application deployed on the cloud and discussed cloud analyst simulator in detailed [9].Author discussed the scheduling policies and their requirement in cloud environment and have also computed comparative study of four common job scheduling algorithms in cloud computing [14]. Khiyaita et al. [4] have surveyed the state-of-the-art of load balancing in cloud computing system. They establish the state of the art load balancing in the cloud computing system, giving a definition of this term, its classification and examples of its implementation in classical distributed systems and in the cloud computing system, key technologies as well as research directions.

\section{PROPOSED WORK}

This study encompassed the comparative analysis of two VM load balancing algorithm as shown in figure 1 . and combination of each with service broker policies to determine overall response time, overall data processing time and total cost. In this study, the Round Robin VM Load balancing policy is taken along with Dynamic Reconfiguring Routing Service broker policy and it is compared with the Active Monitoring Load Balancing policy which is also taken along with Dynamic Reconfiguring Routing Service broker policy and simulation is performed by adjusting parameters to estimate overall response time, data center processing time, response time by region, user base hourly response times, data center hourly average processing times and total cost which is having an impact on performance.

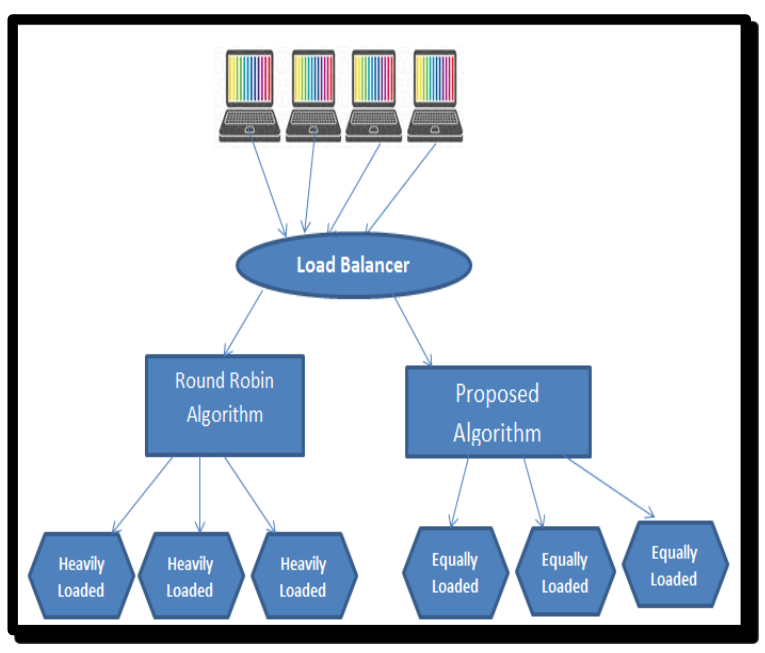

Fig 1: Load Balancing Algorithms Available in Cloud Analyst 


\subsection{Active Tracking Load Balancing Framework}

It is composed of combination of Active Monitoring Load balancing policy as shown in Figure 2. and dynamically reconfigure router service broker policy.

Load balancing policy is utilized by all datacenters in allocating requests to virtual machines. Data center Controller uses virtual machine load balancer to determine which virtual machine should be assigned the next cloudlet for processing. In proposed load balancing policy, load balancer keeps track of number of cloudlets currently being processed by each virtual machine and attempts to balance the active load.

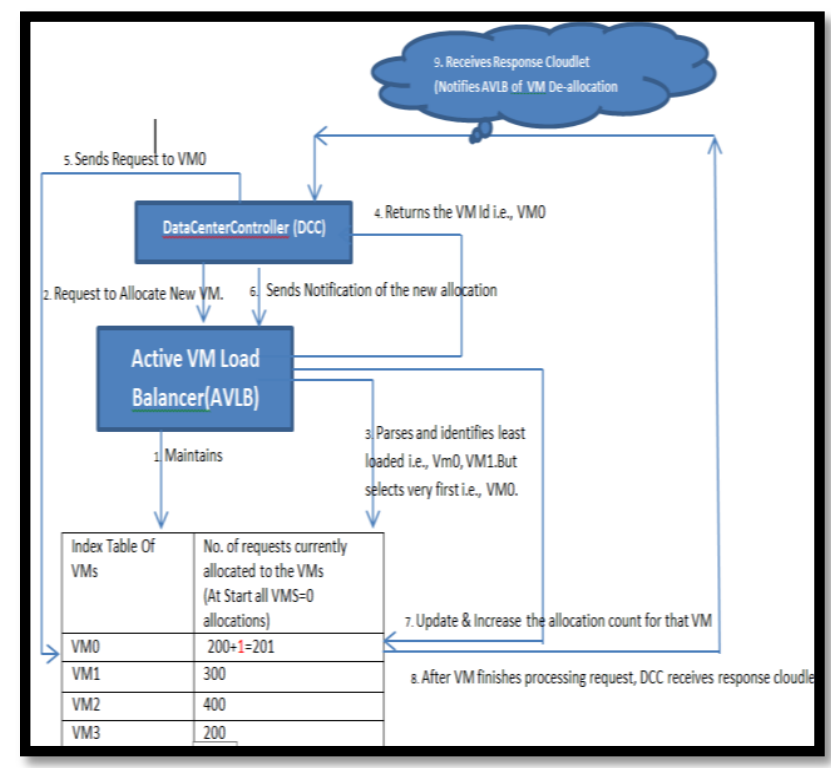

Fig 2: Framework of Active Tracking Load Balancing Algorithm

\subsection{Service Broker Policy}

This is an extended version of Proximity based routing, the routing procedure is similar but the service broker is entrusted with the additional responsibility of scaling the application deployment based on load it is facing [9].

\section{SIMULATION SETUP AND PERFORMANCE ANALYSIS}

This study employs the cloud analyst tool to evaluate the two algorithms namely round robin, active tracking load balancing policies. Simulation setup is done by using six user base identified as UB1, UB2, UB3, UB4, UB5, UB6. Each user base belongs to different region. The four different data centers identified as DC1, DC2, DC3, DC4 having 25, 50 and 75,100 numbers of VMs respectively are also taken.

\subsection{Cloud Analyst [9] [12]}

This study includes the tool which is GUI based and is known as cloud analyst. It is extended version of Cloudsim. It is a toolkit that performs simulation and testing. The central issue with Cloudsim is that in it all the task is needed to be done programmatically or technically. By contrast, Cloud Analyst allows user to do repeated simulations with slight change in parameters smoothly and briskly.
Table1: Various Parameters and their values

\begin{tabular}{|l|l|}
\hline Parameter & Value Passed \\
\hline VM-image size & 10000 \\
\hline VM-memory & $512 \mathrm{MB}$ \\
\hline VM-bandwith & 1000 \\
\hline Service broker policy & Dynamic Service Broker \\
\hline Data center architecture & x86 \\
\hline Data center-OS & Linux \\
\hline Data center -VMM & Xen \\
\hline $\begin{array}{l}\text { Data center-No. of VMs } \\
\text { center-memory per }\end{array}$ & $\begin{array}{l}\text { DC1-25,DC2-50 } \\
\text { DC3-75,DC4-100 }\end{array}$ \\
\hline $\begin{array}{l}\text { Data per } \\
\text { machine }\end{array}$ & $1 \mathrm{~TB}$ \\
\hline $\begin{array}{l}\text { Data center-storage } \\
\text { machine }\end{array}$ & 1000000 \\
\hline $\begin{array}{l}\text { Data center-available bandwith } \\
\text { per machine }\end{array}$ & 10000 \\
\hline Data center -processor speed & Time shared \\
\hline Data center-VM Policy & 1000 \\
\hline User grouping factor & 250 \\
\hline Request grouping factor & \\
\hline Executable instruction length & \\
\hline
\end{tabular}

It is used for constructing and analysis of large scale cloud computing environment. It comprises of three vital menus namely,configure simulation, define internet characteristics and run simulation [7].These menus are used for framing the whole simulation procedure. Simulation setup and analysis of results are carried out for duration of $60 \mathrm{hrs}$. The other parameters are fixed according to Table 1 as shown. The tool estimates the simulation outcome which is based on parameters and shows them in graphical form as shown in Figure 3.

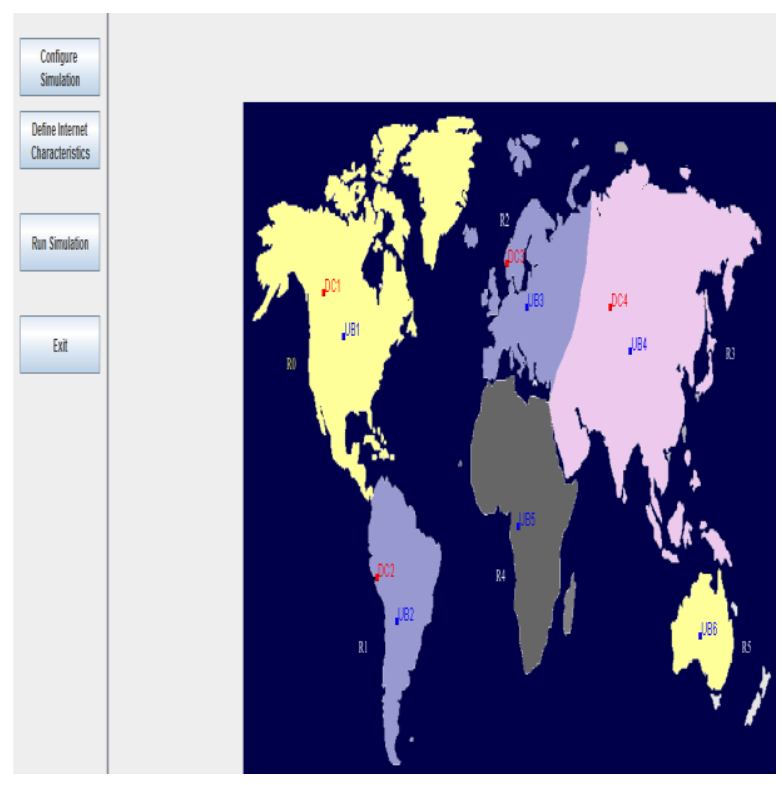

Fig 3: Graphic user interface

In order to perform analysis of various load balancing policies, the adjustment of various parameters of cloud analyst tool has been done. The study includes comparison analysis of two load balancing policies when dynamically reconfigure router service broker policy is taken. We have set the 
parameters for the user base configuration, application deployment configuration, and data center configuration as shown in figure 4 . The location of user bases has been defined in six different regions of the world. We have taken four data centers to handle the request of these users. On DC1 there are $25 \mathrm{VMs}$ allocated, $50 \mathrm{VMs}$ are allocated to DC2, $75 \mathrm{VMs}$ are allocated to DC3 and $100 \mathrm{VMs}$ are allocated to DC4 respectively. The six user bases are also taken.

\subsection{Experimental Results}

After performing the simulation the result computed by cloud analyst is as shown in the following figures. The above defined configuration has been used for each load balancing policy one by one and depending on that the result calculated for the metrics like response time, request processing time and cost in fulfilling the request has been shown. Response time is defined as time taken by an internet application and is defined as interval time between sending of a request and receiving of a response. Request processing time refers to time duration the data centre takes to fulfil the request. The estimated cost is sum total of total virtual machine cost and total data transfer cost.Parameters like average response time, data center service time and total cost of different data centers have taken for analysis. After performing simulation, it is estimated that in case of round robin, there is rise of response time and datacenter processing time whereas in case of proposed active tracking load balancing policy ,there is intense reduction in response time and data center request processing time.

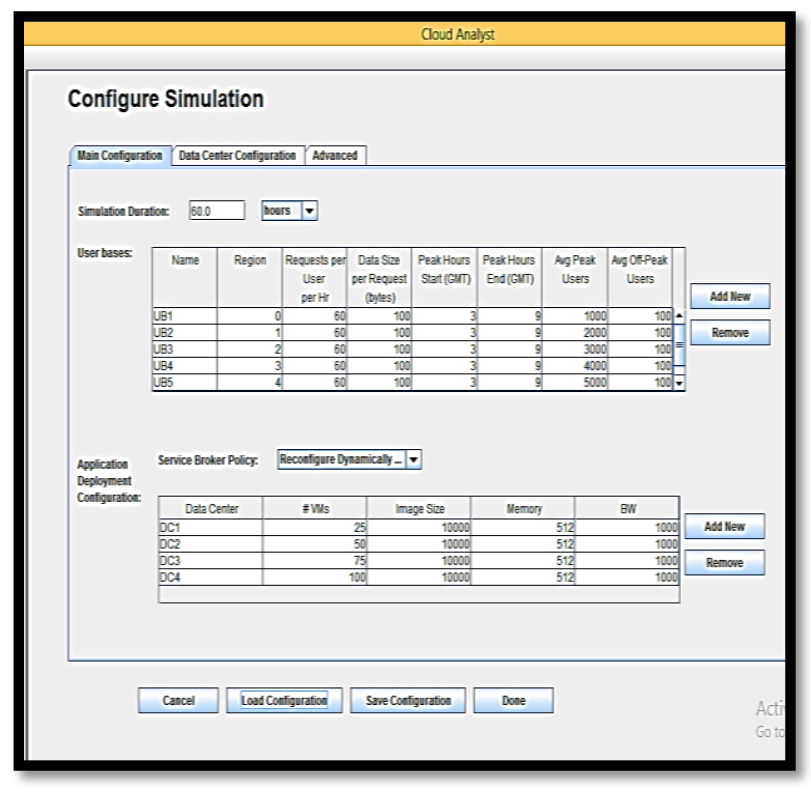

Fig 4: Setting user bases and data center

Case: When Dynamically Reconfiguring Router Service Broker Policy Is Configured.
Table 2: Comparison among load balancing policies (Average response time)

\begin{tabular}{|l|l|l|}
\hline UBS & RR & $\begin{array}{l}\text { PROPOSED } \\
\text { ALGORITHM }\end{array}$ \\
\hline UB1(1000) & 130.63 & 84.97 \\
\hline UB2(2000) & 276.17 & 88.13 \\
\hline UB3(3000) & 162.45 & 89.56 \\
\hline UB4(4000) & 91.29 & 91.26 \\
\hline UB5(5000) & 442.37 & 343.92 \\
\hline UB6(6000) & 1214.97 & 745.02 \\
\hline
\end{tabular}

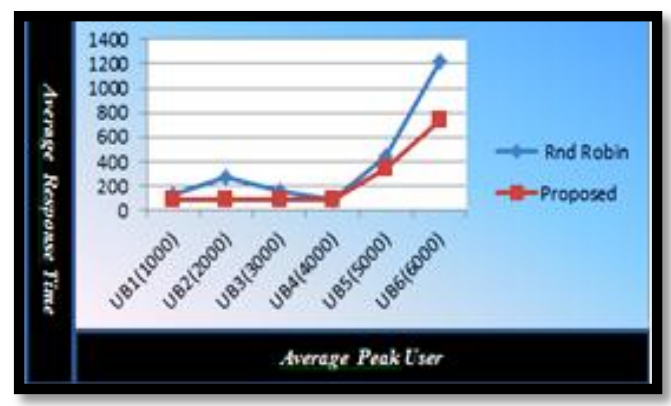

Fig 5: Result showing the average peak users vs. average response time

Table 3: Average data center request servicing time among $L B$ policies

\begin{tabular}{|l|l|l|}
\hline UBS & RR & $\begin{array}{l}\text { PROPOSED } \\
\text { ALGORITHM }\end{array}$ \\
\hline DC1 & 637.93 & 339.24 \\
\hline DC2 & 226.42 & 38.37 \\
\hline DC3 & 128.75 & 42.15 \\
\hline DC4 & 41.7 & 41.68 \\
\hline
\end{tabular}

Figure 6: Analysis by taking average data center serving time and data centers

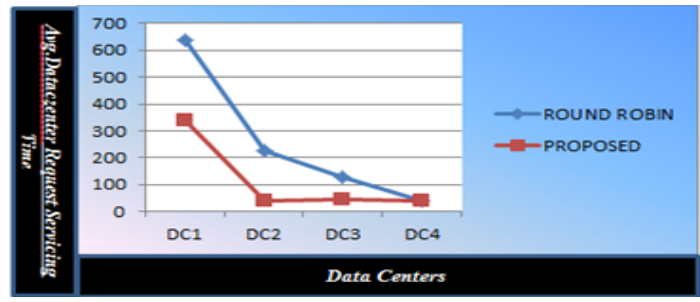

Table 4: Comparison among load balancing policies (Overall response time, Data Center Processing time,

\begin{tabular}{|l|l|l|}
\hline \multicolumn{3}{|c|}{ Total Cost) } \\
\hline PARAMETER & $\begin{array}{l}\text { ROUND } \\
\text { ROBIN }\end{array}$ & $\begin{array}{l}\text { PROPOSED } \\
\text { ALGORITHM }\end{array}$ \\
\hline $\begin{array}{l}\text { OVERALL } \\
\text { RESPONSE } \\
\text { TIME }\end{array}$ & 495.45 & 322.55 \\
\hline $\begin{array}{l}\text { OVERALL } \\
\text { DATA } \\
\text { CENTER } \\
\text { PROCESSING } \\
\text { TIME }\end{array}$ & $\mathbf{2 9 1 . 7 2}$ & 144.37 \\
\hline TOTAL COST & $\$ 2505.47$ & $\$ 2505.48$ \\
\hline
\end{tabular}




\section{CONCLUSION}

While arriving at a conclusion, this study concludes behavior of two different scheduling algorithms has been investigated in cloud environment. Each algorithm is observed and their scheduling criteria like average response time, data center service time and total cost of different data centers are estimated. According to the experiment and comparative analysis the active tracking load balancing algorithm has the best integrate performance. Future work will done by collaborating above algorithm with evolutionary algorithms such as particle swarm optimization algorithm, artificial bee colony algorithm, and diffusion algorithm which is expected to minimize response time in greater amount .

\section{REFERENCES}

[1] Y.Jadeja and K. Modi," Cloud Computing - Concepts, Architecture and Challenges", International Conference on Computing, Electronics and Electrical Technologies [ICCEET] 2012.

[2] Ram Prasad Padhy and PGoutam Prasad Rao ,'Load balancing in cloud computing system", Department of Computer Science and Engineering National Institute of Technology, Rourkela Rourkela-769 008, Orissa, India May, 2011.

[3] M. D. Dikaiakos et al. "Cloud Computing: Distributed Internet Computing for IT and Scientific Research", IEEE Journal of Internet Computing, Vol. 13, No. 5, September/October 2009, pages 10-13.

[4] Khiyaita et al." Load Balancing Cloud Computing: State Of Art", IEEE, 2010.

[5] R. Mata-Toledo and P. Gupta, "Cloud Load Balancing Techniques: A Step towards Green", Journal of Technology Research, Volume No-2 issue- 1, 1-8, 2010.

[6] Alakeel A. M., "A Fuzzy Dynamic Load Balancing Algorithm for Homogenous Distributed Systems", International Journal of Computer Science and Network Security, 10(6), 153-160, 2010.

[7] Jaspreet Kaur, "Comparison Load Balancing Algorithms In A Cloud”, International Journal Of Engineering Research And Applications, 2012.
[8] Martin Randles et al."A Comparative Study into Distributed Load Balancing Algorithms for Cloud Computing", IEEE 24th International Conference on Advanced Information Networking and Applications Workshops, 2010.

[9] Bhathiya, Wickremasinghe,"Cloud Analyst: A Cloud Sim-based Visual Modeller for Analysing Cloud Computing Environments and Applications", IEEE, 2010.

[10] T. Kokilavani , J.J. College of Engineering \& Technology and Research Scholar, Bharathiar University, Tamil Nadu, India" Load Balanced Min-Min Algorithm for Static Meta-Task Scheduling in Grid Computing" International Journal of Computer Applications (0975 - 8887) Volume 20- No.2, April 2011.

[11] P. Goyal et al., "An optimized round robin scheduling algorithm for CPU scheduling", International journal of computer and electrical engineering (IJCEE), vol. 2, No. 7, pp. 2383-2385, December, 2010.

[12] Tanvee Ahmed, Yogendra Singh, "Analytic Study Of Load Balncing Techniques Using Tool Cloud Analyst". International Journal of Engineering Research and Applications. pp. 1027-1030, 2012.

[13] Rich Lee, Bingchiang Jeng, "Load-Balancing Tactics in Cloud", 2011 International Conference on CyberEnabled Distributed Computing and Knowledge Discovery.

[14] Isam Azawi Mohialdeen , "Comparative Study Of Scheduling Algorithms In Cloud Computing Environment", Journal of Computer Science, 2013.

[15] ChaczkoZenon et al., "Availability and Load Balancing in Cloud Computing", International Conference on Computer and Software Modeling IPCSIT, Singapore, 2011.

[16] M.Katyal, A.Mishra," Comparative Study of Load Balancing Algorithms in Cloud Computing", International Journal of Distributed and Cloud Computing, Volume No-1 issue- 2, 2013. 